\title{
A systematic experimental neuropsychological investigation of the functional integrity of working memory circuits in major depression
}

\author{
Oliver Gruber $\cdot$ David Zilles $\cdot$ Jennifer Kennel • \\ Eva Gruber • Peter Falkai
}

Received: 9 July 2010/ Accepted: 25 October 2010/Published online: 10 November 2010

(C) The Author(s) 2010. This article is published with open access at Springerlink.com

\begin{abstract}
Verbal and visuospatial working memory (WM) impairment is a well-documented finding in psychiatric patients suffering from major psychoses such as schizophrenia or bipolar affective disorder. However, in major depression (MDD) the literature on the presence and the extent of WM deficits is inconsistent. The use of a multitude of different WM tasks most of which lack process-specificity may have contributed to these inconsistencies. Eighteen MDD patients and 18 healthy controls matched with regard to age, gender and education were tested using process- and circuit-specific WM tasks for which clear brain-behaviour relationships had been established in prior functional neuroimaging studies. Patients suffering from acute MDD showed a selective impairment in articulatory rehearsal of verbal information in working memory. By contrast, visuospatial WM was unimpaired in this sample. There were no significant correlations between symptom severity and WM performance. These data indicate a dysfunction of a specific verbal WM system in
\end{abstract}

O. Gruber, D. Zilles and J. Kennel have contributed equally.

O. Gruber - D. Zilles · P. Falkai

Center for Translational Research in Systems Neuroscience and Psychiatry, Department of Psychiatry, University Medical

Centre, Georg August University, Goettingen, Germany

J. Kennel · E. Gruber

Department of Psychiatry and Psychotherapy,

Saarland University Hospital, Homburg, Germany

O. Gruber $(\bowtie)$

Department of Psychiatry and Psychotherapy, Georg August University, Von-Siebold-Str. 5, 37075 Goettingen, Germany

e-mail: ogruber@gwdg.de acutely ill patients with MDD. As the observed functional deficit did not correlate with different symptom scores, further, longitudinal studies are required to clarify whether and how this deficit is related to illness acuity and clinical state of MDD patients.

Keywords Major depression · Neurocognition ·

Cognitive endophenotype $\cdot$ Psychosis $\cdot$ Brain imaging

\section{Introduction}

Impaired cognitive functioning in the acute phase of a major depressive disorder is a well-documented finding (for a review see [17]). However, to date there is no consistent pattern of neuropsychological deficits that is characteristic for patients with major depression. Studies seem to indicate a rather diffuse impairment on a broad range of cognitive processes with some more pronounced deficits in specific cognitive domains like executive functioning. Furthermore, the course of these cognitive deficits during the different illness phases is not entirely clear. For example, some authors have argued that there may be a normalisation of working memory functions in remission phases, but that mild deficits may persist in specific tasks that impose additional demands on executive functions.

With respect to working memory performance in major depression, the literature is also inconclusive. Numerous studies reported significant deficits in working memory performance in major depression, particularly in more complex working memory tasks (e.g. [7, 33]), but also in 'simple' digit span [23] or n-back-tasks [18] when investigating patients with moderate to severe depression. Other studies failed to find significant working memory deficits in depressive patients although performance rates of the 
patients were usually reduced when compared to healthy controls (e.g. $[8,32])$. Thus, to date the presence and the extent of working memory disturbances in major depressive patients are largely unclear with some evidence for a possibly greater impairment in tasks with higher or additional (executive) demands.

During the last decade, functional neuroimaging has become a very important tool in current research in the field of biological psychiatry. Besides its role in research on the pathophysiology and therapy of mental disorders, functional neuroimaging has facilitated the development of advanced experimental neuropsychological research methods. State-of-the-art functional neuroimaging relies on the usage of very sophisticated experimental paradigms borrowed from experimental psychology, which allow the targeted investigation of specific cognitive, emotional, motivational or volitional processes that are associated with activation of specific neurofunctional circuits in the human brain.

In a large series of studies investigating healthy controls $[9,10,13,15,16,25]$, psychiatric patients $[14,20-22]$ and patients with focal brain lesions [11], clear brain-behaviour relationships could be established between specific brain circuits and subcomponents of working memory in humans. On the one hand, distinct neural networks have been identified to underlie specific working memory components in healthy subjects. On the other hand, patients with focal brain lesions in brain areas, which had been shown to be activated during performance of specific working memory tasks, exhibited clear task-specific deficits when performing the same task. On the basis of such clearly established and specific brain-behaviour relationships, it becomes possible to attribute particularly isolated behavioural deficits in psychiatric patients to specific dysfunctions of brain circuits and neurofunctional systems identified in prior functional neuroimaging studies [11, 12, 34, 35].

Therefore, the aim of this study was to investigate patients with major depressive disorder using process- and circuit-specific tasks of verbal and visuospatial working memory. In this way, we assessed the functional integrity (in terms of behavioural outcome) of the brain circuits that underlie these different subcomponents of working memory. In the light of previous findings suggesting impaired working memory functioning especially in more severely depressed patients [2, 23], we hypothesised that our sample of acutely depressed patients would also show impaired performance particularly in the verbal domain of working memory. With regard to visuospatial working memory, depressive patients were expected to perform normal as most prior studies failed to find visuospatial working memory deficits in major depression in maintenance tasks without additional executive demands.

\section{Materials and methods}

\section{Subjects}

Twenty-five patients and 47 healthy control subjects were included in this study. The patient group was recruited from inpatients with diagnosis of major depressive disorder (F32 and F33 according to ICD-10 criteria), hospitalised in the Psychiatric Department of the Saarland University Hospital. Healthy comparison subjects were recruited from friends and relatives of the investigators as well as hospital staff. Inclusion criteria for patients were a diagnosis of major depressive disorder and age from 18 to 65 years. Exclusion criteria were acute suicidality, involuntary treatment, current substance abuse, history of brain trauma, diseases with alterations of cerebral metabolism, uncorrected visual or auditory disability and mental retardation. Inclusion criteria for healthy comparison subjects were the same as above plus the absence of any past or present psychiatric disorder. All subjects gave written informed consent before participation, and the study was approved by the local ethics committee.

All subjects underwent a computer-based behavioural experiment described later. The patients' clinical state was assessed on the day of the experiment by using Clinical Global Impression Scale (CGI), Beck Depression Inventory (BDI), Montgomery-Asberg Depression Rating Scale (MADRS).

After a careful matching with respect to age, gender and years of education, statistical analyses were performed on data from 18 patients and 18 healthy controls. All patients except one received antidepressive medication at the time of the testing. Three patients had a combination of two antidepressants (Mirtazapine $n=8$, SSRI $n=3$, tricyclic antidepressants $n=8$, Tranylcypromine $n=1$ ). Both the patient and the healthy control sample consisted of 17 consistent right-handers and one left-hander. For further sample characteristics, see Table 1 .

Table 1 Demographic and clinical characteristics of patients with $\operatorname{MDD}(n=18)$ and healthy control subjects $(n=18)$

\begin{tabular}{llllllll}
\hline & \multicolumn{2}{l}{ Patients } & & \multicolumn{2}{l}{ Controls } & \multirow{2}{*}{$P$} \\
\cline { 2 - 3 } & Mean & SD & & Mean & SD & \\
\hline Demographic factors & & & & & \\
Age (years) & 46.5 & 10.7 & & 44.6 & 11.6 & 0.615 \\
Gender (m/f) & $4 / 14$ & & & $4 / 14$ & & \\
Years of education & 13.8 & 2.4 & & 14.5 & 3.0 & 0.433 \\
Symptom severity & & & & & \\
CGI & 4.28 & 1.02 & & & \\
BDI & 19.94 & 10.66 & & & \\
MADRS & 20.11 & 10.65 & & & \\
\hline
\end{tabular}

CGI Clinical Global Impression, BDI Beck Depression Inventory, $M A D R S$ Montgomery-Asberg depression rating scale 


\section{Experimental design}

Testing was conducted in an experimental neuropsychological laboratory under standardised conditions. The behavioural experiment consisted of four tasks, testing working memory with respect to verbal and visuospatial components, each under single task conditions (i.e. active rehearsal of the sample items) as well as under dual-task conditions (i.e. under articulatory or visuospatial suppression). These different task conditions were conducted in a blocked manner, i.e. each of these specific tasks was repeated 50 times with a short pause after 25 trials. The order of the tasks performed was counterbalanced across subjects within each group.

In the 'verbal rehearsal task', subjects were instructed to vocalise four sample letters internally one time in the presentation period and then to intensely rehearse them throughout the delay period. In the 'non-articulatory phonological maintenance task', subjects were again instructed to subvocalise the four sample letters one time but then to silently count "one, two, three, four, one, two..." and so on during the delay period. This articulatory suppression task has been proven to selectively interfere with verbal working memory $[4,16]$. Subjects were explicitly instructed not to use any visual memory strategies but to concentrate on the mere sound of the letters. In addition, letter case was systematically changed between presentation and response phase to prevent the subjects from using a visual strategy. In the 'visuospatial rehearsal task', subjects got the instruction to repetitively perform overt shifts of attention to the four spatial positions in a $5 \times 5$ matrix that they had to remember. This working memory strategy has been termed a 'visuospatial rehearsal mechanism' by some authors [3]. Finally, in the 'visuospatial pattern maintenance task', subjects were instructed to remember the spatial pattern built by the four sample positions and to perform a visuospatial suppression task during the delay period by following a little moving star with their eyes. This procedure had been proven to selectively interfere with visuospatial working memory $[4,16]$. For each task, there was a practice phase to ensure that participants had understood task instructions.

Stimulus presentation was identical for each of the four task conditions. At the beginning of each trial, a $5 \times 5$ matrix appeared on the monitor with four squares of the matrix filled by different letters, randomly chosen out of a set of eight, in German, phonologically similar letters (B, C, D, E, G, P, T, W). The position of the letters in the matrix was also chosen randomly, with the constraint that no adjacent squares should be filled by letters, to avoid the encoding of two or more squares as a single item. This presentation was visible on the screen for $2 \mathrm{~s}$ followed by a delay period of $3.2 \mathrm{~s}$, in which only the empty matrix could be seen on the monitor together with a little star quickly moving across the screen. Simultaneously, rhythmic $4-\mathrm{kHz}$ tones were presented throughout the delay period with a repetition frequency of $3.3 \mathrm{~Hz}$ to set the pace of the rehearsal or counting. During this interval, subjects had to keep the letters or the spatial positions in mind using the above-described memory strategies. In the following response phase, one letter was presented in a square of the matrix for $2 \mathrm{~s}$. Subjects had to decide whether the probe letter (verbal working memory task) or its position (visuospatial working memory task) matched one of the four target letters or positions, respectively, presented at the beginning. There was an additional 2-s intertrial interval between the single trials during which a fixation cross was presented on the screen. Thus, the total length of a single trial was $9.2 \mathrm{~s}$ resulting in a task duration of about $8 \mathrm{~min}$ for each of the four different tasks.

\section{Statistical analysis}

Statistical analyses were performed using SPSS for Windows 16.0 (SPSS Inc., Chicago, Illinois, USA). Betweengroup comparisons were performed using independent, unpaired, two-tailed $t$ tests using a statistical threshold of $P<0.05$ for each of the four task conditions. Possible relationships between task performance and clinical variables were tested with Pearson correlations, with a significance level of $P<0.05$, corrected for multiple comparisons.

\section{Results}

\section{Working memory performance}

Patients with MDD showed significantly reduced performance specifically in the verbal working memory task requiring the articulatory rehearsal mechanism $(P=0.011$, Cohen's $d=0.91$; see Fig. 1). In this particular task, 8 out of 18 depressive patients performed worse than -1.5 standard deviations compared to the mean of healthy controls. Less pronounced reductions of working memory performance rates were also found for the other tasks which, however, did not reach the level of statistical significance (non-articulatory maintenance of phonological information: $P=0.221$, Cohen's $d=0.415$; visuospatial rehearsal: $P=0.138$, Cohen's $d=0.506$; visuospatial pattern maintenance: $P=0.203$, Cohen's $d=0.433$ ).

Comparing the task conditions, i.e. articulatory or visuospatial rehearsal versus the same task performed under domain-specific (articulatory or visuospatial) suppression, we found a significant effect of articulatory suppression in the control group $(P=0.016)$ in terms of a reduced 


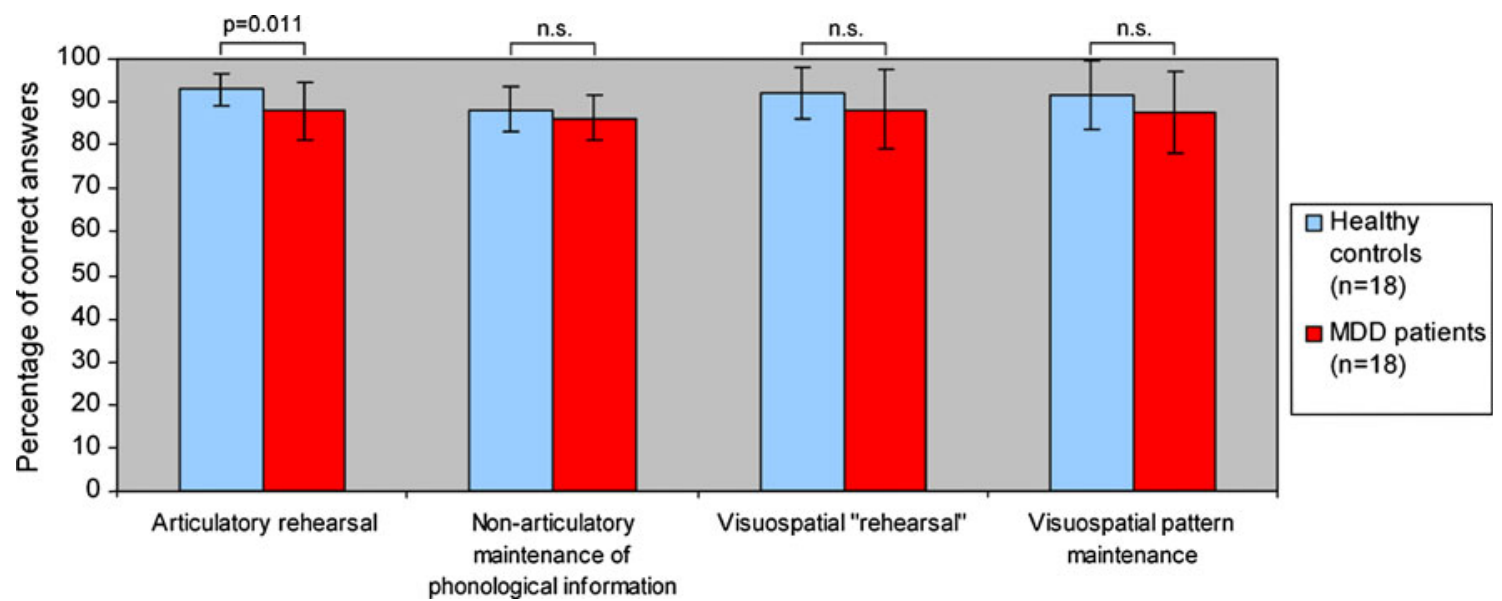

Fig. 1 Group comparisons for four different working memory tasks. Depicted are the mean values and standard deviations for patients with major depressive disorder and healthy controls. Groups were matched with respect to age, gender and years of education

performance in the non-articulatory maintenance task (performed under articulatory suppression) compared to the articulatory rehearsal task. This well-known experimental psychological effect was abolished in the MDD group.

Associations between working memory performance and clinical variables

Correlation analysis was conducted to examine possible associations between working memory task performance and clinical variables (age, gender, years of education and symptom severity measured by BDI, MADRS, CGI). We did not find any significant correlations between performance rates in the different working memory tasks and these demographic and clinical variables (even at an uncorrected level of statistical testing). All correlation coefficients in this analysis were considerably smaller than 0.5 .

\section{Discussion}

This study assessed patients with major depressive disorder with respect to their performance in circuit-specific working memory tasks, which had been shown by prior functional neuroimaging $[9,10,13,15,16]$ and lesion studies [11] to rely on the proper functioning of specific brain systems. We found that patients suffering from acute major depression revealed an isolated deficit in the articulatory rehearsal component of verbal working memory, whereas other components of working memory (non-articulatory maintenance of phonological information and visuospatial working memory) were unimpaired in these patients. Thus, this isolated deficit may be attributed to a specific dysfunction of the mainly left-hemispheric premotor-parietal brain system that has been shown to underlie the articulatory rehearsal mechanism of human verbal working memory [16]. Additional evidence for the selectivity of this deficit of the verbal rehearsal mechanism was obtained by the comparison of different task conditions (i.e. rehearsal vs. suppression) demonstrating that the classical experimental psychological effect of articulatory suppression on verbal working memory task performance, which was also present in the group of healthy subjects in this study, was abolished in the patient group. This further indicates that, in contrast to the significantly reduced capacity of the articulatory mechanism, the capacity of the non-articulatory mechanism of verbal working memory was relatively preserved in MDD patients.

The broader literature on the presence or absence of working memory dysfunctions in patients with major depression is overall inconsistent. Most studies published so far were unable to provide evidence for significant working memory deficits in depressive patients (e.g. [5, 8 , 29, 32]), although in most studies depressive patients showed numerically reduced performance rates in the different working memory tasks. Some of the previous studies provided evidence that depressive patients may exhibit impairments specifically in experimental tasks that either impose higher demands on working memory processes or that require additional executive functions, as is the case in the Wisconsin Card Sorting Test [8], the CANTAB spatial working memory task [33] and in the antisaccade task [24]. Similarly, two studies reported impaired performance in depressed patients in the paced auditory serial addition task (PASAT) which requires working memory and additional arithmetic abilities [7, 26]. In the same studies, patients were unimpaired in digit span tasks [7, 8, 26]. Furthermore, as negative results have been often obtained in out-patient samples with rather mild to moderate depression, it may be possible that working memory deficits may depend on 
symptom severity [2] and may only be observable in severely depressed patients. A recent study performed by Hinkelmann et al. [23] indeed suggests that working memory may be impaired in moderately to severely depressed patients when taken off medication.

At least to some degree, methodological issues are likely to contribute to these inconsistent findings: a problem with traditional neuropsychological studies of working memory dysfunctions in major depression is that most of the tasks used in these studies lack process specificity, i. e. these tasks do not tap on specific cognitive subprocesses that together make up the rather complex architecture of human working memory. The results of this study using more refined and process-specific working memory tasks borrowed from experimental psychology indeed provide evidence for a selective and specific dysfunction of the articulatory rehearsal mechanism of verbal working memory in patients suffering from acute major depression.

This finding is also consistent with previous reports of disturbed verbal fluency in patients with major depression $[19,30]$ as functional magnetic resonance imaging studies have revealed at least a partial overlap of neuroanatomic networks that underlie the articulatory rehearsal mechanism of verbal working memory and verbal fluency. The neural network for the articulatory rehearsal mechanism comprises mainly left-hemispheric regions including Broca's area and the precentral gyrus [16], while verbal fluency tasks elicited activations in a more widespread and bilateral network which, however, also includes posterior parts of the frontal cortex (Brodmann's areas 44 and 45), i.e. Broca's area [1, 31]. Furthermore, fMRI studies in both acutely depressed and remitted patients [27, 28] revealed altered brain activations during verbal fluency task performance. Together, these studies suggest that a dysfunction of a left-hemispheric neural network, which underlies articulatory verbal mechanisms, is involved in both the cognitive disturbances and the pathophysiology of major depressive disorder.

Despite the fact that, in accordance with our initial hypothesis, acutely depressed patients showed deficits in verbal working memory, task performance did not correlate with symptom severity as measured by MADRS, BDI and CGI. Thus, cognitive disturbances and psychopathological symptoms appear to be distinct features of major depressive disorder, and it remains unclear whether and how acuteness of illness and the clinical state of patients with major depression may contribute to the observed cognitive impairment [6]. This underlines the need for longitudinal studies addressing the stability of working memory and other cognitive deficits in the course of major depressive disorder.

In summary, by using process- and circuit-specific working memory tasks, we found a selective deficit of the articulatory rehearsal mechanism in patients with acute major depression. The observed deficit can be attributed to a specific dysfunction of a previously identified lefthemispheric frontoparietal neural network. Future studies should address the question of diagnostic specificity of the pattern of working memory dysfunctions observed in this study for major depression when compared, for example, to bipolar affective disorder. Furthermore, direct functional neuroimaging investigations in patients with major depressive disorder are needed to further assess the pathophysiological changes that may be responsible for the articulatory rehearsal deficit in these patients. In addition, future studies should seek to clarify the influence of acute illness phases and symptom severity on this selective dysfunction of the articulatory rehearsal mechanism in patients with major depression in order to provide evidence for the trait versus state character of the observed working memory deficit. Finally, the investigation of non-affected first-degree relatives of patients with major depressive disorder may also answer the question whether the observed working memory deficit is a heritable and stateindependent endophenotype of major depression.

\section{Conflict of interest None to declare.}

Open Access This article is distributed under the terms of the Creative Commons Attribution Noncommercial License which permits any noncommercial use, distribution, and reproduction in any medium, provided the original author(s) and source are credited.

\section{References}

1. Amunts K, Weiss PH, Mohlberg H, Pieperhoff P, Eickhoff S, Gurd JM, Marshall JC, Shah NJ, Fink GR, Zilles K (2004) Analysis of neural mechanisms underlying verbal fluency in cytoarchitectonically defined stereotaxic space-The roles of Brodmann areas 44 and 45. Neuroimage 22:42-56

2. Austin MP, Mitchell P, Wilhelm K, Parker G, Hickie I, Brodaty H, Chan J, Eyers K, Milic M, Hadzi-Pavlovic D (1999) Cognitive function in depression: a distinct pattern of frontal impairment in melancholia? Psychol Med 29:73-85

3. Awh E, Jonides J, Reuter-Lorenz PA (1998) Rehearsal in spatial working memory. J Exp Psychol Hum Percept Perform 24:780-790

4. Baddeley AD (1986) Working memory. Oxford University Press, Oxford, UK

5. Barch DM, Sheline YI, Csernansky JG, Snyder AZ (2003) Working memory and prefrontal cortex dysfunction: specificity to schizophrenia compared with major depression. Biol Psychiatry 53:376-384

6. Douglas KM, Porter RJ (2009) Longitudinal assessment of neuropsychological function in major depression. Aust N Z J Psychiatry $43: 1105-1117$

7. Egeland J, Sundet K, Rund BR, Asbjornsen A, Hugdahl K, Landro NI, Lund A, Roness A, Stordal KI (2003) Sensitivity and specificity of memory dysfunction in schizophrenia: a comparison with major depression. J Clin Exp Neuropsychol 25:79-93 
8. Grant MM, Thase ME, Sweeney JA (2001) Cognitive disturbance in outpatient depressed younger adults: evidence of modest impairment. Biol Psychiatry 50:35-43

9. Gruber O (2001) Effects of domain-specific interference on brain activation associated with verbal working memory task performance. Cereb Cortex 11:1047-1055

10. Gruber O, Goschke T (2004) Executive control emerging from dynamic interactions between brain systems mediating language, working memory and attentional processes. Acta Psychol 115:105-121

11. Gruber O, Gruber E, Falkai R (2005) Neural correlates of working memory deficits in schizophrenic patients. Ways to establish neurocognitive endophenotypes of psychiatric disorders. Radiologe 45:153-160

12. Gruber O, Gruber E, Falkai P (2006) Articulatory rehearsal in verbal working memory: a possible neurocognitive endophenotype that differentiates between schizophrenia and schizoaffective disorder. Neurosci Lett 405(1-2):24-28

13. Gruber O, Muller T, Falkai P (2007) Dynamic interactions between neural systems underlying different components of verbal working memory. J Neural Transm 114:1047-1050

14. Gruber O, Tost H, Henseler I, Schmael C, Scherk H, Ende G, Ruf M, Falkai P, Rietschel M (2010) Pathological amygdala activation during working memory performance: evidence for a pathophysiological trait marker in bipolar affective disorder. Hum Brain Mapp 31:115-125

15. Gruber O, von Cramon DY (2001) Domain-specific distribution of working memory processes along human prefrontal and parietal cortices: a functional magnetic resonance imaging study. Neurosci Lett 297:29-32

16. Gruber O, von Cramon DY (2003) The functional neuroanatomy of human working memory revisited-Evidence from 3-T fMR1 studies using classical domain-specific interference tasks. Neuroimage 19:797-809

17. Hammar A, Ardal G (2009) Cognitive functioning in major depression-a summary. Front Hum Neurosci 3:26. doi: 10.3389/neuro.09.026.2009

18. Harvey PO (2004) Executive functions and updating of the contents of working memory in unipolar depression. J Psychiatr Res 38:567-576

19. Henry JD, Crawford JR (2005) A meta-analytic review of verbal fluency deficits in depression. J Clin Exp Neuropsychol 27:78-101

20. Henseler I, Falkai P, Gruber O (2009) A systematic fMRI investigation of the brain systems subserving different working memory components in schizophrenia. Eur J Neurosci 30:693-702

21. Henseler I, Falkai P, Gruber O (2010) Disturbed functional connectivity within networks subserving domain-specific subcomponents of working memory in schizophrenia: relation to performance and clinical symptoms. J Psychiatr Res 44(6):364-372
22. Henseler I, Gruber O, Kraft S, Krick C, Reith W, Falkai P (2008) Compensatory hyperactivations As markers of latent working memory dysfunctions in patients with obsessive-compulsive disorder: an fMRI study. J Psychiatry Neurosci 33:209-215

23. Hinkelmann K, Moritz S, Botzenhardt J, Riedesel K, Wiedemann K, Kellner M, Otte C (2009) Cognitive Impairment in major depression: association with salivary cortisol. Biol Psychiatry 66:879-885

24. Hutton SB, Ettinger U (2006) The antisaccade task as a research tool in psychopathology: a critical review. Psychophysiology 43:302-313

25. Koelsch S, Schulze K, Sammler D, Fritz T, Müller K, Gruber O (2009) Functional architecture of verbal and tonal working memory: an FMRI study. Hum Brain Mapp 30(3):859-873

26. Landro NI, Stiles TC, Sletvold H (2001) Neuropsychological function in nonpsychotic unipolar major depression. Neuropsychiatry Neuropsychol Behav Neurol 14:233-240

27. Okada G, Okamoto Y, Morinobu S, Yamawaki S, Yokota N (2003) Attenuated left prefrontal activation during a verbal fluency task in patients with depression. Neuropsychobiology 47:21-26

28. Okada G, Okamoto Y, Yamashita H, Ueda K, Takami H, Yamawaki S (2009) Attenuated prefrontal activation during a verbal fluency task in remitted major depression. Psychiatry Clin Neurosci 63:423-425

29. Purcell R, Maruff P, Kyrios M, Pantelis C (1997) Neuropsychological function in young patients with unipolar major depression. Psychol Med 27:1277-1285

30. Ravnkilde B, Videbech P, Clemmensen K, Egander A, Rasmussen NA, Rosenberg R (2002) Cognitive deficits in major depression. Scand J Psychol 43:239-251

31. Ravnkilde B, Videbech P, Rosenberg R, Gjedde A, Gade A (2002) Putative tests of frontal lobe function: a PET-study of brain activation during Stroop's test and verbal fluency. J Clin Exp Neuropsychol 24:534-547

32. Sweeney JA, Kmiec JA, Kupfer DJ (2000) Neuropsychologic impairments in bipolar and unipolar mood disorders on the CANTAB neurocognitive battery. Biol Psychiatry 48:674-684

33. Tavares JVT, Clark L, Cannon DM, Erickson K, Drevets WC, Sahakian BJ (2007) Distinct profiles of neurocognitive function in unmedicated unipolar depression and bipolar II depression. Biol Psychiatry 62:917-924

34. Zilles D, Burke S, Schneider-Axmann T, Falkai P, Gruber O (2009) Diagnosis-specific effect of familial loading on verbal working memory in schizophrenia. Eur Arch Psychiatry Clin Neurosci 259:309-315

35. Zilles D, Gruber E, Falkai P, Gruber O (2010) Patients with schizophrenia show deficits of working memory maintenance components in circuit-specific tasks. Eur Arch Psychiatry Clin Neurosci 260:519-529 